\title{
Investigation of HNF-1B as a diagnostic biomarker for pancreatic ductal adenocarcinoma
}

\author{
Michelle X. Yang ${ }^{1,7^{*}}$, Ryan F. Coates ${ }^{1}$, Abiy Ambaye ${ }^{1}$, Juli-Anne Gardner ${ }^{1}$, Richard Zubarick ${ }^{2}$, Yuan Gao ${ }^{3}$, \\ Joan Skelly ${ }^{4}$, James G. Liư ${ }^{5}$ and Mari Mino-Kenudson ${ }^{6}$
}

\begin{abstract}
Background: Diagnosing pancreatic ductal adenocarcinoma (PDAC) in the setting of metastasis with an unknown primary remains very challenging due to the lack of specific biomarkers. HNF-1B has been characterized as an important transcription factor for pancreatic development and was reported as a biomarker for clear cell subtype of PDAC.

Methods: To investigate the diagnostic role of HNF-1B for PDAC, we used tissue microarray (TMA) and immunohistochemistry (IHC) to characterize HNF-1B expression in a large cohort of carcinomas, including 127 primary PDACs, 47 biliary adenocarcinomas, 17 metastatic PDACs, and 231 non-pancreaticobiliary carcinomas.

Results: HNF-1B was expressed in 107 of 127 (84.3\%) of PDACs, 13 of 15 (86.7\%) of cholangiocarcinomas, 13 of 18 (72\%) of ampullary carcinomas, and 13 of 14 (92.9\%) of gallbladder adenocarcinomas. Notably, HNF-1B was expressed in 16 of 17 (94.1\%) of metastatic PDACs. Among the non-pancreaticobiliary cancers, HNF-1B was expressed in $\sim 77 \%$ clear cell carcinomas of the kidney and ovarian clear cell carcinomas. Gastroesophageal, lung, and prostate adenocarcinomas occasionally expressed HNF-1B in up to 37\% cases. HNF-1B was completely negative in hepatocellular, colorectal, breast, and lung squamous cell carcinomas. The sensitivity, specificity, positive predictive value, negative predictive value, and accuracy of HNF-1B for primary pancreaticobiliary carcinoma is $84,68,66,85$, and $75 \%$, respectively. HNF-1B expression was not significantly associated with overall survival in patients with PDAC, but tumor size $\geq 2 \mathrm{~cm}$ and high tumor grade were significantly associated with worse overall survival in multivariate analyses.
\end{abstract}

Conclusions: HNF-1B may be used in surgical pathology to aid the diagnosis of metastatic pancreatic and biliary carcinoma with a panel of other markers to exclude lung, kidney, prostate, and Müllerian origins.

Keywords: HNF-1B, Pancreatic, Pancreaticobiliary, Adenocarcinoma, Tissue microarray, Immunohistochemistry

\section{Background}

Pancreatic ductal adenocarcinoma (PDAC) consists of approximately $85 \%$ of cancers arising in the pancreas, and is one of the most lethal malignancies in the world. Despite new generations of neoadjuvant and adjuvant therapies, the 5-year overall survival rate remains less than 5\%, and patients with PDAC often present with metastatic disease of an unknown primary $[1,2]$. An accurate diagnosis of PDAC on biopsy specimens remains

\footnotetext{
* Correspondence: michelle.yang@umassmemorial.org

'Department of Pathology and Laboratory Medicine, University of Vermont

Medical Center, 111 Colchester Avenue, Burlington, VT, USA

${ }^{7}$ Present address: Department of Pathology, University of Massachusetts

Medical Center, 1 Innovation Drive, Worcester, MA 01605, USA

Full list of author information is available at the end of the article
}

challenging due to the lack of specific biomarkers $[3,4]$. Investigating additional markers to improve the diagnosis of PDAC is of paramount important in daily practice for surgical pathologists.

Hepatocyte nuclear factor 1B (HNF-1B) has been well-characterized as one of the transcription factors involved in the early development of liver, pancreas, and kidney [5-7]. In animal models, HNF-1B gene was required for the morphogenesis of both ventral and dorsal pancreatic buds $[8,9]$. In human subjects, mutations in HNF-1B caused severe pancreatic agenesis or hypoplasia, maturity-onset diabetes of the young (MODY) type 5, multicystic renal dysplasia, and hepatobiliary tract and Müllerian tract abnormalities [10-14]. In human adenocarcinomas,

(C) The Author(s). 2018 Open Access This article is distributed under the terms of the Creative Commons Attribution 4.0 International License (http://creativecommons.org/licenses/by/4.0/), which permits unrestricted use, distribution, and 
HNF-1B was highly expressed in ovarian clear cell carcinomas and has been recognized as a useful molecular biomarker for this entity [15-17]. Interestingly, a recent study showed that PDAC with clear cell morphology strongly expressed HNF-1B, in contrast to the conventional type PDAC with only weak $(61 \%)$ to moderate $(24 \%)$ staining [18]. Due to the essential role of HNF-1B in pancreatic development, we hypothesized that HNF-1B was expressed in all cancers arising from the pancreatic ductal epithelium regardless of the histomorphology, and its expression may serve as a diagnostic marker of these cancers. Using immunohistochemistry (IHC) and tissue microarray (TMA), we investigated HNF-1B protein expression in 127 primary PDACs, 47 biliary tract adenocarcinomas, 17 metastatic PDACs, and 231 common non-pancreaticobiliary carcinomas, and calculated its sensitivity and specificity. The utility of HNF-1B to aid the diagnosis of pancreaticobiliary adenocarcinoma was discussed.

\section{Methods}

\section{Study population}

A total of 127 primary PDAC resections and 17 known metastatic PDACs were retrospectively retrieved from formalin fixed paraffin embedded (FFPE) blocks. Among the 127 primary PDACs, 10 cases received neoadjuvant therapy and 112 cases had negative resection margins (R0). A total of 85 cases had complete survival data with at least 2 years of follow-up after resection. Among the 17 metastatic PDACs, metastatic sites included liver $(N=13)$, celiac lymph nodes $(N=2)$, peritoneum $(N=1)$, and bone $(N=1)$. In addition, 47 adenocarcinomas from the biliary tract and 231 non-pancreaticobiliary carcinomas that morphologically mimic PDAC (mimickers) were also evaluated for comparison, including those of the ampulla, intrahepatic and extrahepatic biliary tract, gallbladder, colorectal, esophagus, stomach, hepatocellular carcinoma, lung (both adenocarcinoma and squamous cell carcinoma), bladder (urothelial carcinoma), breast (ductal and lobular), kidney (mainly clear cell carcinoma), prostate, ovarian surface epithelial, and endometrial. This study was approved by our Institutional Review Board (IRB \# 17-0009).

\section{Histological evaluation and tissue microarray (TMA) construction}

All tumor slides of the 127 primary PDAC resections were reviewed, and the size of the tumor, tumor (pT) and nodal $(\mathrm{pN})$ stages, tumor grade, tumor morphology (cytoplasmic clearing), lymphovascular invasion (LVI), perineural invasion (PNI), and resection margin status were extracted from the electronic pathologic record. The death status were extracted from the Tumor Registry data set. The final stage of PDAC was diagnosed in accordance with the American Joint Committee on Cancer (AJCC), 7th edition [19]. Two-millimeter core TMAs were constructed with two cores each from the FFPE tumor tissue or adjacent non-neoplastic pancreas (as control) of primary PDAC resections or 278 non-pancreatic cancers (Beecher Instruments Inc., Sun Prairie, WI). Eleven of 17 metastatic PDACs were also included in the duplicated 2-mm core TMA, and the remaining 6 metastatic PDACs were biopsies that were mounted onto individual slides.

\section{Immunohistochemistry (IHC)}

Polyclonal anti-HNF-1B (Sigma, St. Louis, MO, HPA002083, 1:200 dilutions) was validated in non-neoplastic pancreatic tissue sections. Antigen retrieval was obtained for HNF-1B in $\mathrm{H} 1$ buffer (Leica Biosystems, Buffalo Grove, IL) for $10 \mathrm{~min}$, All IHCs were performed in Leica BOND-III automated IHC stainer. Localization of staining - nuclear, cytoplasmic and/or membranous - was recorded in each case, and the case was recorded as positive if any amount of tumor cells had any pattern of HNF-1B expression. A two-tier scale was applied to all positive cases for HNF-1B: "strong", if the stain was clearly visualized at $20 \times$ low magnification, and "weak", if the stain was clearly visualized at $100 \times$ magnification with less intensity.

As comparison, a monoclonal antibody (Abnova, clone CL0374, 1:200 dilution) was validated under the same conditions mentioned above and showed nuclear reactivity in normal pancreatic ductal epithelium, but not in the acinar cells or islet cells, which was consistent with the result of polyclonal antibody. All cancer TMAs were investigated with the HNF-1B expression using the polyclonal antibody.

\section{Statistical analysis}

All demographic and clinicopathological measures were screened for ranges and appropriate codes. Eighty five of the 127 primary PDAC patients had complete demographic and clinicopathological measures and had survival data with at least 2 years of follow-up after the resection. For these 85 patients, descriptive statistics for these measures were calculated. The association between HNF-1B protein expression and clinicopathological features was examined using $2 \times 2$ contingency tables with Fisher's extract tests. Univariate Cox proportional hazard function models were used to examine the association of each of the clinicopathological measures with overall survival. The significant measures were included in a stepwise modeling procedure to determine a final model. The expression rate of HNF-1B in PDAC was compared to the rate in each of the other cancer types using Fisher's exact tests. The sensitivity, specificity and other measures of HNF-1B in classifying pancreaticobiliary and non-pancreaticobiliary carcinomas were calculated with 95\% confidence intervals. All statistical analyses were conducted using SAS 9.3 software (SAS Institute, Cary NC). Statistical significant was determined by $P<.05$. 


\section{Results}

Demographics and clinicopathologic features of PDAC

Among the 85 primary PDAC patients with complete clinicopathologic data, the mean age was 65 years $(\mathrm{SD}=10)$, 55 were male $(65 \%)$ (Table 1$)$. Sixty eight $(80 \%)$ had positive HNF-1B expression. Fisher's exact tests found no significant associations between HNF-1B expression and the clinicopathologic parameters. In univariate analysis, only tumor size $\geq 2 \mathrm{~cm}(P=.03)$ and high tumor grade $(P=.02)$ were significantly associated with worse overall survival. HNF-1B protein expression did not demonstrate significant association with the overall survival. In multivariate analysis, tumor size $\geq 2 \mathrm{~cm}(P=.03)$ and high tumor grade $(P=.02)$ remained significantly associated with worse overall survival (Table 2).

\section{HNF-1B expression in non-neoplastic pancreatic and biliary epithelium}

In non-neoplastic adult pancreas, HNF-1B was expressed in the ductal epithelium and centroacinar ductal cells with predominant nuclear and faint cytoplasmic staining (Fig. 1a). Since HNF-1B is also a transcription factor for liver development, we investigated HNF-1B immunostaining in normal liver and gallbladder. Interestingly, HNF-1B showed

Table 1 Demographics and clinicopathological parameters in patients with PDAC

\begin{tabular}{|c|c|}
\hline Variables & $\operatorname{PDAC}(N=85)$ \\
\hline Age: Mean \pm SD (years) & $65 \pm 10$ \\
\hline \multicolumn{2}{|l|}{ Gender } \\
\hline Male & $55(65 \%)$ \\
\hline Female & $30(35 \%)$ \\
\hline \multicolumn{2}{|l|}{ Tumor size } \\
\hline$\leq 2 \mathrm{~cm}$ & $18(21 \%)$ \\
\hline$>2 \mathrm{~cm}$ & $67(79 \%)$ \\
\hline \multicolumn{2}{|l|}{ Tumor location } \\
\hline Head & $63(74 \%)$ \\
\hline Tail & $22(26 \%)$ \\
\hline LN metastasis & $54(64 \%)$ \\
\hline LVI & $40(48 \%)$ \\
\hline $\mathrm{PNI}$ & $61(72 \%)$ \\
\hline No neoadjuvant & $75(88 \%)$ \\
\hline \multicolumn{2}{|l|}{ Stage } \\
\hline Stage I, II & $24(28 \%)$ \\
\hline Stage III, IV & $61(72 \%)$ \\
\hline \multicolumn{2}{|l|}{ Tumor grade } \\
\hline Low grade $(1 / 2)$ & $56(66 \%)$ \\
\hline High grade (3) & $29(34 \%)$ \\
\hline
\end{tabular}

Abbreviations: PDAC pancreatic ductal adenocarcinoma; $S D$ standard deviation, LVI lymphovascular invasion; $L N$ lymph node, $P N I$ perineural invasion
Table 2 Overall survival and clinicopathological variables in 85 patients with PDAC

\begin{tabular}{lll}
\hline Variables & Hazard ratio $(95 \% \mathrm{Cl})$ & $p$-value \\
\hline Univariate analysis & $0.98(0.59-1.62)$ & .94 \\
Age $(<65$ vs $\geq 65)$ & $1.13(0.68-1.88)$ & .65 \\
Gender (male vs. female) & $2.11(1.07-4.16)$ & .03 \\
Tumor size (< 2 cm vs. $\geq 2 \mathrm{~cm})$ & $0.74(0.44-1.26)$ & .28 \\
Stage (I/II vs. III/IV) & $1.84(1.11-3.05)$ & .02 \\
Grade (low vs. high) & $1.18(0.70-2.01)$ & .54 \\
PNI & $1.46(0.90-2.40)$ & .13 \\
LVI & $1.31(0.77-2.21)$ & .32 \\
LN metastasis & $1.34(0.70-2.57)$ & .38 \\
HNF-1B (negative vs. positive) & $1.14(0.41-1.14)$ & .80 \\
No neoadjuvant & & $\mathbf{. 0 3}$ \\
Multivariate analysis & $2.10(1.06-4.16)$ & $\mathbf{. 0 2}$ \\
Tumor size $(<2$ cm vs. $\geq 2 \mathrm{~cm})$ & $1.83(1.10-3.06)$ & \\
Grade (low vs. high) & &
\end{tabular}

Abbreviations: PDAC, pancreatic ductal adenocarcinoma; VS, versus; $S D$ standard deviation, LVI lymphovascular invasion; LN lymph node, PNI perineural invasion Bold: Statistically significant

predominant nuclear staining in the gallbladder epithelium (Fig. 1b), in contrast to a predominant cytoplasmic staining in non-neoplastic intrahepatic ductal and extrahepatic ductal epithelium (Fig. 1c).

Morphological variation of primary and metastatic PDAC In a previous study, Kim et al. reported that HNF-1B was strongly expressed in PDAC with cytoplasmic clearing and only weakly to moderately expressed in PDAC with conventional histomorphology. ${ }^{18}$ We reviewed the morphology specifically the cytoplasmic clearing for all the PDAC cases in this cohort and separated them into 3 categories: 1 ) Conventional- if $>95 \%$ of tumors cells demonstrated no cytoplasmic clearing (Fig. 2a); 2) Prominent clearing- if $>75 \%$ tumor cells demonstrated cytoplasmic clearing (Fig. 2b); and 3) mixed features- if 5-75\% tumor cells demonstrated cytoplasmic clearing (Fig. 2c). Among the 127 primary PDACs, 84 cases $(66.1 \%)$ were conventional PDAC, and 43 cases $(33.9 \%)$ showed variable cytoplasmic clearing in the tumor cells, including 10 cases $(7.9 \%)$ with prominent clearing, and 33 cases (26\%) with mixed features. In 17 metastatic PDACs, 1 case showed prominent cytoplasmic clearing, 2 cases had mixed features, and the remaining showed conventional morphology.

\section{Variable expression pattern of HNF-1B in primary and metastatic PDAC}

Although HNF-1B was characterized as a nuclear transcription factor, HNF-1B was expressed in a total of 107 (84.3\%) PDACs with multiple staining patterns, including 


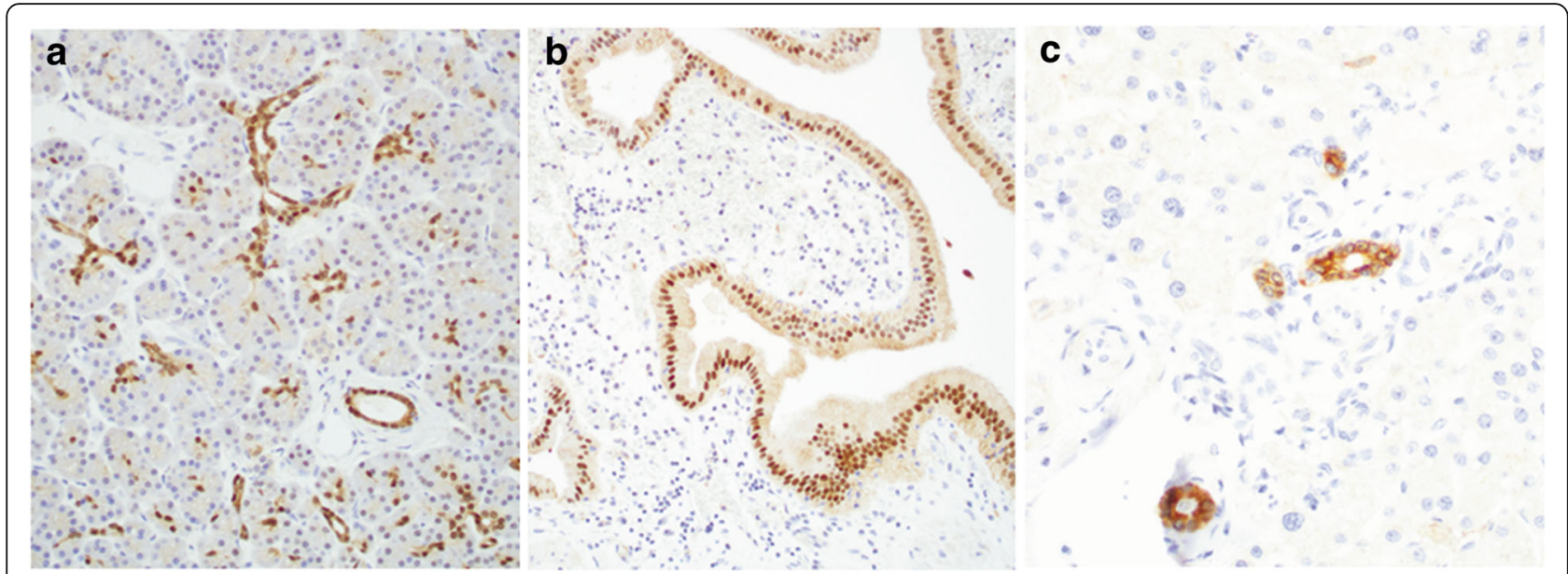

Fig. 1 HNF-1B expression pattern by IHC in non-neoplastic pancreaticobiliary epithelium, including (a) Pancreas, (b) Gallbladder, and (c) intrahepatic bile duct. Original magnification, 200x

cytoplasmic staining in 64 (59.8\%) cases (Fig. 3a), nuclear staining in $32(29.9 \%)$ cases (Fig. 3b), nuclear and cytoplasmic staining in $6(5.6 \%)$ cases (Fig. 3c), and cytoplasmic and membranous staining in $5(4.7 \%)$ cases (Fig. 3d). Among the 107 HNF-1B positive PDAC cases, 90 cases (70.9\%) showed strong staining, while 17 (13.4\%) showed weak staining pattern. There was no significant association of HNF-1B staining pattern or intensity with cytoplasmic clearing of the tumor cells.

Sixteen of 17 (94.1\%) metastatic PDACs were positive for HNF-1B, including 13 cases (76.5\%) with strong staining and 3 cases (17.6\%) showed weak staining. Interestingly, 12 metastatic PDACs (70.6\%) showed predominantly nuclear immunoreactivity with or without cytoplasmic staining (Fig. 3e, f).

\section{HNF-1B expression in non-pancreatic carcinomas}

In order to investigate whether HNF-1B protein expression was restricted to the adenocarcinomas of pancreatic ductal origin, HNF-1B IHC was performed on a total of 278 common carcinomas from other organ systems, including cholangiocarcinoma (intrahepatic and extrahepatic biliary tracts), ampullary region, gallbladder, colorectum, distal esophagus, stomach, hepatocellular, lung adenocarcinoma, lung squamous cell carcinoma, breast, prostate, ovary, uterus, bladder urothelial, and kidney.

Not surprisingly, HNF-1B was strongly immunoreactive with nuclear and/or cytoplasmic patterns in 13 of 15 (86.7\%) intrahepatic and extrahepatic cholangiocarcinomas, 13 of 18 (72.2\%) ampullary adenocarcinomas, and 13 of 14 (92.9\%) gallbladder adenocarcinomas. In addition, strong nuclear immunoreactivity of HNF-1B was observed in 16 of 21 (76.1\%) clear cell carcinomas of the kidney, 10 of 14 (71.4\%) ovarian clear cell carcinomas, 6 of 24 (25\%) lung adenocarcinomas, and 4 of 15 (26.7\%) prostate adenocarcinomas. Weak nuclear with or without cytoplasmic HNF-1B expression was seen in 3 of 11 (27.3\%) esophageal adenocarcinomas, 9 of $14(64.3 \%)$ stomach adenocarcinomas, 7 of 18 bladder urothelial (38.9\%), and 10 of 21 (42.8\%) non-clear cell type Müllerian carcinomas. HNF-1B was completely negative in all colorectal cancer, breast cancer, hepatocellular carcinoma, and lung squamous cell

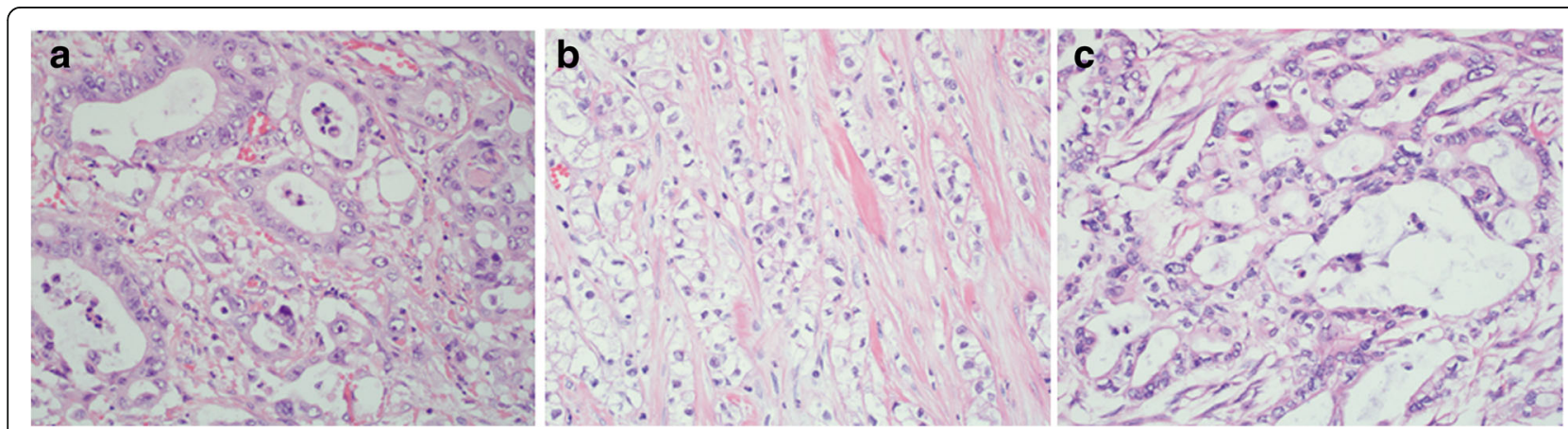

Fig. 2 Histomorphological variation of PDAC on hematoxylin and eosin (HE) staining, including (a) conventional, (b) clear cell variant, and (c) mixed features. Original magnification, 400x 


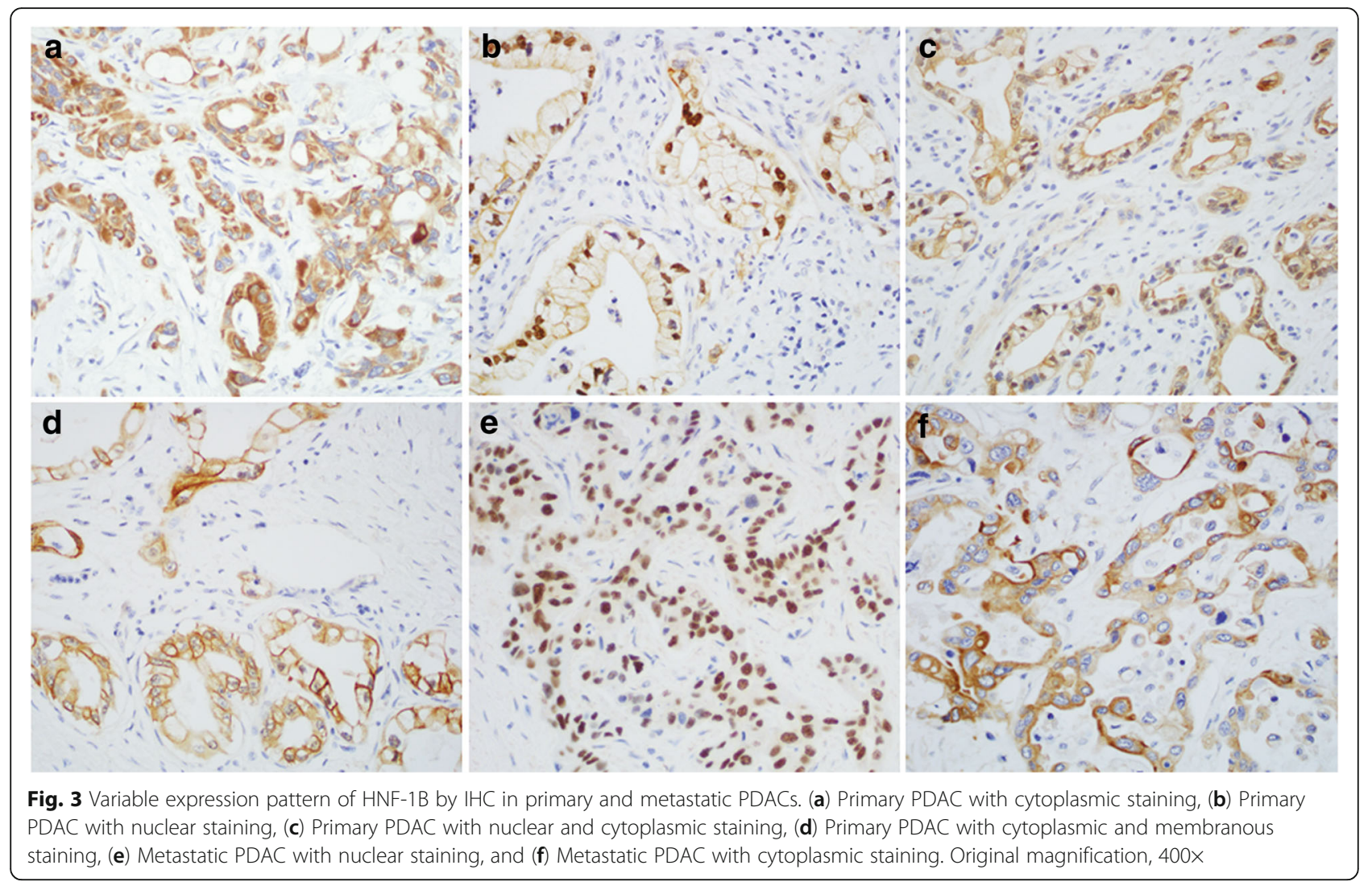

Table 3 Immunoreactivity of HNF-1B in PDAC and non-pancreatic carcinomas

\begin{tabular}{|c|c|c|c|c|}
\hline Cancer type & Total No. & HNF-1B + & Stain pattern & Fisher's exact $p$-value \\
\hline Pancreatic primary & 127 & $107(84.3 \%)$ & $C, N, M$ & ref \\
\hline Cholangiocarcinoma & 15 & $13(86.7 \%)$ & $C, N, M$ & .81 \\
\hline Ampullary & 18 & $13(72 \%)$ & $C, N, M$ & .21 \\
\hline Gallbladder & 14 & $13(92.9 \%)$ & $C, N, M$ & .69 \\
\hline Colorectal & 39 & 0 & - & $<.001$ \\
\hline Hepatocellular & 20 & 0 & - & $<.001$ \\
\hline Esophagus & 11 & $3(27.3 \%)$ & $C, N$ & $<.001$ \\
\hline Stomach & 14 & $9(64.3 \%)$ & $\mathrm{C}, \mathrm{N}$ & .13 \\
\hline Lung adenocarcinoma & 24 & $6(25 \%)$ & $C, N$ & $<.001$ \\
\hline Lung Squamous cell & 10 & 0 & - & $<.001$ \\
\hline Breast & 20 & 0 & - & $<.001$ \\
\hline Bladder & 18 & 7 (38.9\%) & $C, N$ & $<.001$ \\
\hline Prostate & 15 & $4(26.7 \%)$ & $C, N$ & $<.001$ \\
\hline Kidney & 21 & $16(76.2 \%)$ & $\mathrm{N}$ & .35 \\
\hline Ovary & 18 & $14(77.8 \%)$ & $\mathrm{N}, \mathrm{C}$ & .50 \\
\hline Uterus & 21 & $10(47.6 \%)$ & $\mathrm{N}, \mathrm{C}$ & $<.001$ \\
\hline Metastatic PDAC & 17 & $16(94.1 \%)$ & $\mathrm{N}, \mathrm{C}$ & \\
\hline Total No. & 422 & & & \\
\hline
\end{tabular}


carcinoma (Table 3). Fisher's exact tests showed significantly lower HNF-1B expression rate in the colorectal, hepatocellular, esophageal, lung, breast, bladder, prostate, and uterine carcinomas $(P<.001$, Table 3$)$.

\section{Sensitivity and specificity of HNF-1B in pancreaticobiliary carcinomas}

Since we observed that HNF-1B was expressed in the majority of carcinomas arising from the pancreatic and biliary epithelium, we calculated the sensitivity, specificity, positive predictive value, negative predictive value, and accuracy with $95 \%$ confidence interval $(95 \% \mathrm{CI})$ of HNF-1B for all primary pancreaticobiliary carcinomas in comparison to non-pancreaticobiliary carcinomas. The results were summarized in Table 4. Overall, HNF-1B showed high sensitivity (84\%) and high negative predictive value $(85 \%)$ for primary pancreaticobiliary carcinomas with moderate specificity and accuracy (68 and 75\%, respectively).

\section{Discussion}

We investigated the protein expression pattern of HNF-1B in 127 primary PDACs, 47 biliary adenocarcinomas, 17 metastatic PDACs, and 231 other common carcinomas that may mimic PDAC. HNF-1B was highly expressed in adenocarcinomas along the pancreaticobiliary tract, including PDAC, intrahepatic and extrahepatic cholangiocarcinomas, ampullary adenocarcinomas, and gallbladder adenocarcinomas with nuclear and/or cytoplasmic staining pattern. Importantly, HNF-1B expression was expressed in $94.1 \%$ of metastatic PDAC with predominantly nuclear staining.

KRAS mutation is a frequent molecular abnormality that is identified in up to $90 \%$ of PDACs [20]. Interestingly, a recent study showed that mutated KRAS can induce abnormal regulations of pancreatic transcription factors including HNF-1B, which in turn causes abnormal cell growth and proliferation that leads to pancreatic cancer [21]. These findings were consistent with the fundamental pathophysiological role of HNF-1B in the pancreaticobiliary system. The other group has also reported cytoplasmic and/or nuclear expression of HNF-1B in PDAC [18]. Although transcription factors are translated in the cytoplasm, they are generally translocated into

Table 4 Sensitivity and specificity of HNF-1B in pancreaticobiliary carcinomas

\begin{tabular}{lll}
\hline Measure & Proportion & $95 \% \mathrm{Cl}$ \\
\hline Sensitivity & 0.84 & $(0.79,0.90)$ \\
Specificity & 0.68 & $(0.62,0.74)$ \\
Positive Predictive Value & 0.66 & $(0.60,0.73)$ \\
Negative Predictive Value & 0.85 & $(0.80,0.90)$ \\
Accuracy & 0.75 & $(0.71,0.79)$ \\
\hline
\end{tabular}

Abbreviation: $\mathrm{Cl}$ confidence interval the nucleus to regulate downstream target genes in active physiological state. During inactive regulation or with aberrantly excessive expression, transcription factors may form complex with other proteins and remain in the cytoplasm and/or cell membrane. This might explain why HNF-1B showed variable nuclear, cytoplasmic and/or membranous staining patterns in PDAC and other carcinomas.

Among non-pancreaticobiliary carcinomas, clear cell carcinomas of the kidney showed predominantly nuclear HNF-1B expression, while Müllerian origin, including ovarian and endometrial clear cell carcinoma showed nuclear and/or cytoplasmic HNF-1B expression. The expression of HNF-1B in carcinomas of kidney and Müllerian origin also indicates its pathophysiological role in these organs. Interestingly, silencing of HNF-1B expression secondary to promoter methylation appears to promote disease progression via epithelial-to-mesenchymal transition in both prostate and ovarian cancers [22]. In contrast to the association of HNF-1B with poor prognosis in PDAC observed by Kim et al. [18], HNF-1B seemed to have tumor-suppressor role in both prostate and kidney cancers [22, 23]. Thus, a biological role(s) of HNF-1B as well as the significance of its aberrant cytoplasmic and membranous expressions in different types of cancer needs to be investigated in the future.

\section{Conclusions}

Our data suggested that HNF-1B may serve as a useful diagnostic biomarker for tumors of the pancreaticobiliary origin with high sensitivity and negative predictive value, but moderate specificity and accuracy for these tumors. Since HNF-1B can also be expressed less frequently in variable tumors of the non-pancreaticobiliary origin, especially of the kidney, Müllerian tract, lung, gastroesophageal, bladder, and prostate carcinomas, the concurrent use of other markers such as TTF-1, PAX-8, WT-1, CAIX, NKX3.1, p40, and PSA as a panel to rule out other organ primaries, and correlation with imaging studies and/or endoscopic findings are important to refine the diagnosis.

\section{Abbreviations}

CAIX: Carbonic anhydrase 9; FFPE: Formalin fixed paraffin embedded; HE: Hematoxylin and eosin; HNF-1B: Hepatocyte nuclear factor 1B; IHC: Immunohistochemistry; MODY: Maturity-onset diabetes of the young; NKX3.1: NK3 Homeobox 1; PAX-8: Paired box gene 8; PDAC: Pancreatic ductal adenocarcinoma; PSA: Prostate specific antigen; SD: Standard deviation; TMA: Tissue microarray; TTF-1: Thyroid transcription factor 1; WT1: Wilms' tumor 1

\section{Acknowledgements}

We are very grateful for the technical support from Valerie M Cortright, Jeannette M Mitchell, and Alexa Buskey of the Department of Pathology and Laboratory Medicine, University of Vermont Medical Center, Burlington, VT 05401. 


\section{Funding}

This work was supported by funding from the University of Vermont Medical Group Research Award to M.X.Y for the proposal entitled "delineating tumors of pancreatic origin" (MG171).

\section{Availability of data and materials}

All data generated or analyzed during this study are available from the corresponding author on reasonable request.

\section{Authors' contributions}

MXY initiated the design of this project. AA, AG, YG, RZ, JGL, and MMK all participated in the design of this study. RFC collected clinical data. JS performed the statistics. MXY and RFC interpreted the protein expression and wrote the manuscript. All authors read, revised and approved the final manuscript.

\section{Ethics approval and consent to participate}

This study was approved by the Institutional Review Board (IRB \#CHRMS 17-0009) at the University of Vermont Medical Center.

\section{Consent for publication}

Not applicable.

\section{Competing interests}

The authors declare that they have no competing interests.

\section{Publisher's Note}

Springer Nature remains neutral with regard to jurisdictional claims in published maps and institutional affiliations.

\section{Author details}

'Department of Pathology and Laboratory Medicine, University of Vermont Medical Center, 111 Colchester Avenue, Burlington, VT, USA.

${ }^{2}$ Gastroenterology, University of Vermont Medical Center, 111 Colchester Avenue, Burlington, VT, USA. ${ }^{3}$ Department of Gastrointestinal Surgery, Nanjing Medical University affiliated Changzhou 2nd People's Hospital, Changzhou, Jiangsu, China. ${ }^{4}$ University of Vermont Medical Biostatistics Department, Burlington, VT, USA. ${ }^{5}$ Applied Pathology Systems, Worcester, MA, USA. ${ }^{6}$ Department of Pathology, Massachusetts General Hospital, Boston, MA, USA. ${ }^{7}$ Present address: Department of Pathology, University of Massachusetts Medical Center, 1 Innovation Drive, Worcester, MA 01605, USA

Received: 11 April 2018 Accepted: 19 July 2018

Published online: 27 July 2018

\section{References}

1. Siegel RD, Naishadham D, Jemal A. Cancer statistics, 2012. CA Cancer J Clin. 2012:62(1):10-29.

2. $\mathrm{Yu}$ J, Blackford AL, Molin MD, Wolfgang CL, Goggins M. Time to progression of pancreatic ductal adenocarcinoma from low-to-high tumour stages. Gut. 2015:64(11):1783-9.

3. Malaguarnera G, Giordano M, Paladina I, et al. Markers of bile duct tumors. World J Gastrointest Oncol. 2011:3(4):49-59.

4. Lin F, Chen ZE, Wang HL. Utility of immunohistochemistry in the pancreatobiliary tract. Arch Pathol Lab Med. 2015;139(1):24-38.

5. Bach I, Mattei MG, Cereghini S, Yaniv M. Two members of an HNF1 homeoprotein family are expressed in human liver. Nucleic Acids Res. 1991; 19(13):3553-9.

6. Cereghini S, Ott MO, Power S, Maury M. Expression patterns of vHNF1 and HNF1 homeoproteins in early postimplantation embryos suggest distinct and sequential developmental roles. Development. 1992;116(3):783-97.

7. Reichert M, Rustgi AK. Pancreatic ductal cells in development, regeneration, and neoplasia. J Clin Invest. 2011;121(12):4572-8

8. Haumaitre C, Barbacci E, Jenny M, Ott MO, Gradwohl G, Cereghini S. Lack of TCF2/VHNF1 in mice leads to pancreas agenesis. Proc Natl Acad Sci U S A. 2005;102(5):1490-5.

9. De Vas MG, Kopp JL, Heloit C, Sander M, Cereghini S, Haumaitre C. Hnf1b controls pancreas morphogenesis and the generation of Ngn3+ endocrine progenitors. Development. 2015;142(5):871-82.
10. Body-Bechou D, Loget P, D'Herve D, et al. TCF2/HNF-1 beta mutations: 3 cases of fetal severe pancreatic agenesis or hypoplasia and multicystic renal dysplasia. Prenat Diagn. 2014;34(1):90-3.

11. Haumaitre C, Fabre M, Cormier S, Baumann C, Delezoide AL, Cereghini S. Severe pancreas hypoplasia and multicystic renal dysplasia in two human fetuses carrying novel HNF1beta/MODY5 mutations. Hum Mol Genet. 2006; 15(15):2363-75.

12. Kitanaka S, Miki Y, Hayachi $Y$, Igarashi T. Promoter-specific repression of hepatocyte nuclear factor (HNF)-1 beta and HNF-1 alpha transcriptional activity by an HNF-1 beta missense mutant associated with type 5 maturityonset diabetes of the young with hepatic and biliary manifestations. J Clin Endocrinol Metab. 2004;89(3):1369-78

13. Haldorsen IS, Vesterhus $M$, Raeder $H$, et al. Lack of pancreatic body and tail in HNF1B mutation carriers. Diabet Med. 2008;25(7):782-7.

14. Teo AK, Lau HH, Valdez IA, et al. Early developmental perturbations in a human stem cell model of MODY5/HNF1B pancreatic hypoplasia. Stem Cell Reports. 2016;6(3):357-67.

15. Tsuchiya A, Sakamoto M, Yasuda J, et al. Expression profiling in ovarian clear cell carcinoma: identification of hepatocyte nuclear factor-1 beta as a molecular marker and a possible molecular target for therapy of ovarian clear cell carcinoma. Am J Pathol. 2003;163(6):2503-12.

16. Huang W, Cheng XM, Ji J, Zhang J, Li Q. The application value of HNF-1 beta transcription factor in the diagnosis of ovarian clear cell carcinoma. Int $J$ Gynecol Pathol. 2016:35(1):66-71.

17. Kato N, Sasou S, Motoyama T. Expression of hepatocyte nuclear factor-1beta (HNF- 1beta) in clear cell tumors and endometriosis of the ovary. Mod Pathol. 2006;19(1):83-9.

18. Kim L, Liao J, Zhang $M$, et al. Clear cell carcinoma of the pancreas: histopathologic features and a unique biomarker: hepatocyte nuclear factor1 beta. Mod Pathol. 2008;21(9):1075-83.

19. Edge SB, Compton CC. The American joint committee on Cancer: the 7th edition of the AJCC cancer staging manual and the future of TNM. Ann Surg Oncol. 2010;17(6):1471-4.

20. Miglio U, Oldani A, Mezzapelle R, et al. KRAS mutational analysis in ductal adenocarcinoma of the pancreas and its clinical significance. Pathol Res Pract. 2014;210(5):307-11.

21. Naqvi AAT, Hasan GM, Hassan MI. Investigating the role of transcription factors of pancreas development in pancreatic cancer. Pancreatology. 2018; 18(2):184-90.

22. Ross-Adams H, Ball S, Lawrenson K, et al. HNF1B variants associate with promoter methylation and regulate gene networks activated in prostate and ovarian cancer. Oncotarget. 2016;7(46):74734-46.

23. Buchner A, Castro M, Hennig A, et al. Downregulation of HNF-1B in renal cell carcinoma is associated with tumor progression and poor prognosis. Urology. 2010;76(2):507 e6-11.

Ready to submit your research? Choose BMC and benefit from

- fast, convenient online submission

- thorough peer review by experienced researchers in your field

- rapid publication on acceptance

- support for research data, including large and complex data types

- gold Open Access which fosters wider collaboration and increased citations

- maximum visibility for your research: over $100 \mathrm{M}$ website views per year

At BMC, research is always in progress.

Learn more biomedcentral.com/submissions 